\title{
The Effect of Psychological Adaptation on Prevention Behavior of College Students during the Coronavirus Disease(COVID-19): A Moderated Mediating Model
}

\author{
Jun Ren \\ Mianyang Normal University: Mianyang Teachers' College \\ Caihong Yang \\ Central China Normal University \\ Fei Huang \\ HUST: Huazhong University of Science and Technology \\ Yan Zhang ( $\sim$ zy1981927@126.com) \\ HUST: Huazhong University of Science and Technology \\ Hui Shi \\ Beijing Chao-Yang Hospital: Beijing Chaoyang Hospital
}

\section{Research Article}

Keywords: Coronavirus Disease 2019, colleges students, psychological adaptation, preventive behavior, risk cognition, stress coping, moderated mediating model

Posted Date: June 2nd, 2021

DOI: https://doi.org/10.21203/rs.3.rs-551884/v1

License: (c) (i) This work is licensed under a Creative Commons Attribution 4.0 International License.

Read Full License 


\section{Abstract}

Background: Coronavirus disease 2019 is an infectious disease. From January 2020 to now, the disease is still a public health event.

Aims: Our aim in the present study was to examine the effects of psychological adaptation on preventive behavior, the mediating effect of risk cognition and the moderating effect of stress coping.

Methods: We took colleges students in China as the survey subject; data were collected during the peak period of the COVID-19.

Results: Psychological adaptation had a significant negative predictive effect on preventive behavior. Risk cognition had a partial mediating effect between psychological adaptation and preventive behavior. Stress coping plays a moderating effect in the first half of the mediating process of psychological adaptation $\rightarrow$ risk cognition $\rightarrow$ preventive behavior, and individuals with high stress coping are more vulnerable to the impact of psychological adaptation on risk cognition.

Conclusions: The impact of psychological adaptation on preventive behavior can occur through the mediation variable of risk cognition. Reducing the risk cognition of college students helps to enhance the positive predictive effect of psychological adaptation on preventive behavior. Moreover, stress coping plays a moderating effect of the mediation model. improving the stress coping of colleges and universities students helps to better increase the negative predict effect of psychological adaptation on risk cognition.

\section{Introduction}

Lifestyle choice is an important determinant of personal health [1]. Individual behavior, especially the choice of preventive measures, plays a key role in the spread of respiratory disease [2]. The effective preventive behaviors include health education, vaccination, antiviral treatment and avoiding exposure to virus [3].

Preventive behavior is essential for the control of epidemic situation and prevention of infection, and it is very effective [4]. The study found that through a series of preventive actions, such as the adoption of new methods of health education in communities and schools, the infectious diseases in Southeast Asia has been effectively prevented [5]. Health belief education can increase residents' susceptibility to infectious diseases [6]. The study found that individual and community level factors were significantly associated with Ebola prevention behavior [7]. Fear of assessing COVID-19 was significantly associated with the preventive behavior of COVID-19 [8]. Cancer information seeking (CISE) is significantly associated with cancer prevention behavior [9]. Compared with urban residents, rural residents are less likely to have preventive behaviors, they hold negative attitude towards the effectiveness of preventive behaviors, and their information evaluation skills are lower [10]. In the absence of vaccines or drugs, nondrug interventions are the most important coping strategies based on community interventions, such as 
distance between people, wearing masks, isolation and good personal hygiene (hand washing) - all of which have been proven to reduce the spread of this seemingly unstoppable global impact of natural disasters [11]. The performance of preventive behavior increased with the increase of educational level and age [12]. Skin cancer related preventive behaviors can reduce the risk of skin cancer [13].

Psychological adaptation refers to that when the external environment changes, the subject makes psychological activities and behavior through the self-regulation system, and is a positive internal growth process in which individuals redefine the way they want to be realized or start a new hope [14]. It has been found that strengthening psychological capital contributes to better psychological adaptation [15]. It is found that the health-related quality of life in high acceptance group and high dependence group is significantly higher than that in control group [16]. There is a negative correlation between psychological adaptation and emotional distress [17]. A 10-year follow-up study found that there was a significant negative correlation between physical adaptation and depression [18].

Some researchers explored the relationship between tooth health and psychological adaptation. The results showed that the participants with higher degree of psychological adaptation were more likely to seek preventive care, and both behavioral and psychological cultural adaptation could predict oral health outcomes [19]. Individuals with higher resilience are more likely to have adaptive preventive behavior [20]. The study found that fear of not being able to cope with or unable to adapt to the environment, namely cognitive vulnerability perceptions, was significantly negatively correlated with preventive behavior [21]. In view of this, we propose hypothesis 1: psychological adaptation can positively predict preventive behavior.

Respondents who know more about Ebola are more likely to take preventive actions against Ebola virus, while those who think they are at high risk of Ebola infection are much less likely to engage in Ebola prevention [7]. Because the actual risk of infection is usually uncertain, individual behavior is affected by perceived risk. Low risk cognition may reduce the use of preventive measures (behavior), and there is a bidirectional correlation between risk perception and behavior; with the increase of risk awareness, preventive behavior also increases [2]. Disease cognition is related to individual seeking help and prevention behavior [22]. There is a significant correlation between climate risk perception and farmers' adaptation behavior [23].

According to the protection motivation theory (PMT), there are two main perception processes: the first is "risk assessment", that is, a person assesses the possibility and potential damage of a threat to the things he cherishes without changing his behavior. This cognitive process produces a specific perception of risk; the second process is "adaptation assessment", in which a person assesses his or her ability to avoid being threatened and the cost of such action. The result of this cognitive process is a specific perceptual adaptability [24]. If we only pay attention to the risk and do not make psychological adaptation changes, there may be some avoidable maladjustment reactions such as rejecting risk [25]. Therefore, we speculate that since psychological adaptation is an adaptive assessment of risk, high psychological 
adaptation means low risk cognition. In view of this, we propose hypothesis 2: risk cognition has a mediating effect between psychological adaptation and preventive behavior.

Stress coping can be defined as the individual's psychological resources when dealing with stress or adverse situations; stress coping includes not only actions, including behaviors (cognition and emotion), and personal assessment of the situation [26]. Some research found that stress coping can affect the individual's response to stress, and then affect the results of mental health [27]. Positive stress coping reduces cancer-related negative behavioral reactions [28]. Stress coping is significantly correlated with risk cognition [29]. Stress vulnerability and good stress coping are the corresponding concepts. Some researchers have studied the relationship between stress vulnerability and risk cognition and other psychological factors in earthquake disasters, and found that people's stress vulnerability is significantly positively related to their cognition of earthquake risk [30]. Therefore, we speculate that compared with high stress coping individuals, low stress coping individuals have higher risk perception, so their psychological adaptation has a stronger negative predictive effect on their risk cognition. In view of this, we propose hypothesis 3 : stress coping moderates the relationship between psychological adaptation and $b$ risk cognition.

In conclusion, this study mainly discusses the impact of psychological adaptation on prevention behavior of college students during the peak period of epidemic, and then explores the mediating role of risk cognition and the moderating role of stress coping on the mediating process. The hypothetical model is shown in Fig. 1.

\section{Research Method}

\subsection{Subjects and procedures}

The questionnaire collected data on www.wjx.cn during February 17-19, 2020. The subjects were 1374 universities students in China. There were 683 males and 691 females with an average age of $20.31 \pm$ 2.80 years.

\subsection{Research material}

\subsubsection{Psychological adaptation questionnaire}

Based on literature analysis [31], expert discussion, we developed a psychological adaptation questionnaire composed of four items, which were integrated into one major category. The questionnaire adopts $1-5$ five-point scoring method. The total internal consistency coefficient of the questionnaire is 0.732 .

\subsubsection{Risk cognition questionnaire}

Based on literature analysis [32], expert discussion, we developed a risk cognition questionnaire composed of three items, which were integrated into one category. The questionnaire adopts 1-5 five- 
point scoring method. The total internal consistency coefficient of the questionnaire was 0.370 .

\subsubsection{Stress coping questionnaire}

Based on literature analysis [33], expert discussion, we developed a stress coping questionnaire composed of five items, which were integrated into two categorie. The questionnaire adopts a scoring method of 1-5 points. The internal consistency a coefficients of stress susceptibility and stress regulation were 0.531 and 0.605 .

\subsubsection{Preventive behavior questionnaire}

Based on literature analysis [7], expert discussion, we developed a preventive behavior questionnaire composed of five items, which were integrated into one category, with five factors in total. The questionnaire was scored from 1 to 5 points. The total internal consistency coefficient of the questionnaire was 0.811 .

\subsection{Statistical analyses}

SPSS 25.0 and Process Syntax were used to perform the regression analysis and construct the predicted mediation model.

\section{Result}

\subsection{Common method bias analysis}

Haman single factor method was used to test the common method bias. The results of data analysis showed that four characteristic roots were larger than 1, and the first common factor could explain $32.127 \%$ of the total variation, which was far lower than the recommended critical value of $40 \%$.

\subsection{Correlation analysis}

We conducted correlation analysis on the variables. See Table 1 for details.

Table 1

Correlation Analysis of each variable

\begin{tabular}{|lllllll|}
\hline & M & SD & $\mathbf{1}$ & $\mathbf{2}$ & $\mathbf{3}$ & $\mathbf{4}$ \\
\hline 1. Preventive behavior & 3.76 & 0.78 & 1 & & & \\
\hline 2. Risk cognition & 3.87 & 0.58 & $-0.24^{* \star}$ & 1 & & \\
\hline 3. Psychological adaptation & 3.65 & 0.77 & $0.68^{\star \star}$ & $-0.17^{\star \star}$ & 1 & \\
\hline 4. Stress coping & 3.28 & 0.60 & $0.30^{\star \star}$ & $-0.11^{\star \star}$ & $0.35^{\star \star}$ & 1 \\
\hline Note: the data have been standardized. ${ }^{* \star} P<0.01$. & & & \\
\hline
\end{tabular}




\subsection{Mediating effect test}

The total effect of psychological adaptation on preventive behavior was tested, and the path coefficient was positively significant $(\beta=0.681, t=34.433, S E=0.020, p<0.001)$. After the mediating variables, risk cognition, was added to the model,the results show that psychological adaptation can significantly negatively predict risk cognition; psychological adaptation have a significant positive predictive effect on preventive behavior and risk cognition have a significant negative predictive effect on preventive behavior. This indicates that risk cognition plays a partial mediating role in the influence of psychological adaptation on preventive behavior (see Fig. 2).

\section{4 moderated mediating effect test}

The interaction between psychological adaptation and stress coping had significant predictive effect on risk cognition $(\beta=0.050, t=1.996 \square S E=0.025 \square p=0.046)$. After stress coping was put into model 7 , the interaction between psychological adaptation and stress coping had significant predictive effect on preventive behavior $(\beta=0.040 \square t=1.996 \square p=0.046)$, indicating that stress coping plays a moderating role in psychological adaptation on preventive behavior prediction (see Fig. 3).

Simple slope analysis showed that compared the subjects with the high stress coping subjects, simple slope $=-0.090, t=-2.203 \rrbracket S E=0.041 \square p=0.028$, the psychological adaptation of subjects with low stress coping has more negative predictive effect on preventive behavior, simple slope $=-0.190 \rrbracket t=-5.390 \rrbracket S E=$ $0.035 \unrhd p<0.001$ (see Fig. 4).

\section{Discussion}

\subsection{The influence of psychological adaptation on preventive behavior}

The results of correlation analysis showed that there was a significant positive correlation between psychological adaptation and preventive behavior. For this result, previous studies have found that the participants with higher degree of psychological adaptation were more likely to seek preventive care, and both behavioral and psychological cultural adaptation could predict oral health outcomes [19]. The possible reason for these phenomena is that Psychological adaptation is a way for individuals to adapt to the environment, and preventive behavior is also an adaptive behavior of individuals from the perspective of evolution. Therefore, it is not difficult to explain the phenomenon that psychological adaptation is significantly related to preventive behavior.

Regression analysis showed that psychological adaptation could significantly positively predict preventive behavior, which was inconsistent with the first hypothesis. The possible reason for these 
phenomena is that individuals with high psychological adaptation are more likely to accept changes in the environment, face unknown risks, and are more likely to make decisions to prepare for the future.

\subsection{Mediating effect of risk cognition on the relationship between psychological adaptation on preventive behavior}

Pearson correlation analysis showed that there was a significant negative correlation between risk cognition and preventive behavior. Previous researchers showed that respondents who know more about Ebola are more likely to take preventive actions against Ebola virus, while those who think they are at high risk of Ebola infection are much less likely to engage in Ebola prevention [7]; with the increase of risk cognition, prevention behavior also increases [2]; risk perception of disease is related to individual seeking help and prevention behavior [22]; there is a significant correlation between climate risk perception and farmers' adaptation behavior [23]. Correlation analysis also showed that there is a significant negative correlation between risk cognition and psychological adaptation. Previous researchers showed that

The mediating effect test showed that psychological adaptation could negatively predict risk cognition and positively predict preventive behavior; risk cognition could negatively predict preventive behavior. This result is consistent with the second hypothesis. This indicates that risk cognition can weaken and buffer the positive effect of psychological adaptation on preventive behavior.

\subsection{Moderating effect of stress coping on mediating effect}

Therefore, we explored whether stress coping has a moderating effect on the above mediating effects. Pearson correlation analysis results showed that stress coping and psychological adaptation are significantly positively correlated. Pearson correlation analysis results also showed that stress coping is significantly negatively correlated with risk cognition. Previous studies have found that stress coping is significantly correlated with risk cognition [29]; people's stress vulnerability is significantly related to their cognition of earthquake risk [30].

The moderating effect showed that stress coping moderates the first half of the path from psychological adaptation to risk cognition to preventive behavior. Compared the subjects with the high stress coping subjects, the psychological adaptation of subjects with low stress coping has more negative predictive effect on preventive behavior. This result is consistent with the third hypothesis.

\section{Conclusion}

The impact of psychological adaptation on preventive behavior can occur through the mediation variable of risk cognition. Reducing the risk cognition of college students helps to enhance the positive predictive effect of psychological adaptation on preventive behavior. Moreover, stress coping plays a moderating effect of the mediation model. improving the stress coping of colleges and universities students helps to better increase the negative predict effect of psychological adaptation on risk cognition. Therefore, after a large sample survey, it was found that the psychological stress of young people in Hubei was significantly different at different stages of the epidemic: Corresponding suggestions were finally 
proposed, schools and units of all levels and types should strengthen the mental health knowledge education, allowing the participants to be more aware of their mental state, and can self-adjust in a suitable manner for them.

\section{Declarations}

\section{Ethics approval and consent to participate}

The study was designed in accordance with the tenets of the Declaration of Helsinki. Approval from the ethical authority of School of Educational Science, Huazhong University of Science and Technology was granted. Confidentiality and the statement confirming informed consent was managed by placing anonymous coding of one self-report questionnaires.

\section{Consent for publication}

This manuscript has not been published or presented elsewhere in part or in entirety. All study participants provided informed consent, and the study design was approved by the appropriate ethics review boards. All the authors have approved the manuscript and agree with submission to your esteemed journal. There are no conflicts of interest to declare.

\section{Availability of data and material}

We can provide all of the data and material to the article.

\section{Competing interests}

There are no conflicts of interest to declare.

\section{Funding}

This study was supported and granted by the "Fundamental Research Funds for the Central Universities: HUST (2019WKZDJC006)" to YZ.

\section{Authors' contributions}

Conceived and designed the questionnaire: ZY, SH, HF. Recruitment and payment of participants: ZY, SH. Analyzed the data: $\mathrm{YCH}, \mathrm{HF}$. Wrote and revised the paper: RJ, YCH, HF.

\section{Acknowledgements}

We thank all participants for their time and interest as well as the editor and reviewers for their valuable feedback.

\section{References}


1. Dong, W.Y., et al., Effects of China's urban basic health insurance on preventive care service utilization and health behaviors: Evidence from the China Health and Nutrition Survey. Plos One, 2018. 13(12).

2. Aerts, C., et al., Understanding the role of disease knowledge and risk perception in shaping preventive behavior for selected vector-borne diseases in Guyana. Plos Neglected Tropical Diseases, 2020. 14(4).

3. Yamamoto, S., et al., [Epidemiology of cholangiocarcinoma]. Nippon Rinsho Japanese Journal of Clinical Medicine, 2015. 73 Suppl 3: p. 615-617.

4. Srithongklang, W., et al., Effect of Educational Intervention Based on the Health Belief Model and Self-Efficacy in Promoting Preventive Behaviors in a Cholangiocarcinoma Screening Group. Journal of Cancer Education, 2019. 34(6): p. 1173-1180.

5. Padchasuwan, N., et al., Modifying Health Behavior for Liver Fluke and Cholangiocarcinoma Prevention with the Health Belief Model and Social Support Theory. Asian Pac J Cancer Prev, 2016. 17(8): p. 3721-3725.

6. Kaewpitoon, S.J., et al., Primary Care Intervention to Prevent and Control Cholangiocarcinoma: Lesson from Nakhon Ratchasima, Thailand. Chotmaihet Thangphaet, 2016. 99(Suppl. 7): p. 8-15.

7. Tenkorang, E.Y., Effect of knowledge and perceptions of risks on Ebola-preventive behaviours in Ghana. International Health, 2018. 10(3): p. 202-210.

8. Ahorsu, D.K., et al., Associations Between Fear of COVID-19, Mental Health, and Preventive Behaviours Across Pregnant Women and Husbands: An Actor-Partner Interdependence Modelling. International Journal of Mental Health and Addiction, 2020.

9. Bhandari, D., et al., Cancer information seeking and scanning behavior among Nepalese migrants in Japan and its association with preventive behavior. Plos One, 2020. 15(6).

10. Chen, X.W. and H.L. Chen, Differences in Preventive Behaviors of COVID-19 between Urban and Rural Residents: Lessons Learned from A Cross-Sectional Study in China. International Journal of Environmental Research and Public Health, 2020. 17(12).

11. Cvetkovic, V.M., et al., Preparedness and Preventive Behaviors for a Pandemic Disaster Caused by COVID-19 in Serbia. International Journal of Environmental Research and Public Health, 2020. 17(11).

12. Haque, A., et al., Comparing the preventive behavior of medical students and physicians in the era ofCOVID-19: Novel medical problems demand novel curricular interventions. Biochemistry and Molecular Biology Education, 2020.

13. Jeihooni, A.K. and M. Moradi, The Effect of Educational Intervention Based on PRECEDE Model on Promoting Skin Cancer Preventive Behaviors in High School Students. Journal of Cancer Education, 2019. 34(4): p. 796-802.

14. Griesemer, I., et al., Psychological Adaptation to Diagnostic Genomic Sequencing Results: The Role of Hope Fulfillment. Health Psychology, 2019. 38(6): p. 527-535. 
15. Aparicio, M.G., et al., Profiles of Psychological Adaptation Outcomes at Discharge From Spinal Cord Injury Inpatient Rehabilitation. Archives of Physical Medicine and Rehabilitation, 2020. 101(3): p. 401-411.

16. Kamody, R.C., et al., Psychological Flexibility Among Youth with Type 1 Diabetes: Relating Patterns of Acceptance, Adherence, and Stress to Adaptation. Behavioral Medicine, 2018. 44(4): p. 271-279.

17. Lacomba-Trejo, L., et al., Questionnaire on adaptation to type 1 diabetes among children and its relationship to psychological disorders. Revista Latino-Americana De Enfermagem, 2018. 26.

18. Timko, C., et al., Children of parents with unipolar depression: A comparison of stably remitted, partially remitted, and nonremitted parents and nondepressed controls. Child Psychiatry \& Human Development, 2002. 32(3): p. 165-185.

19. Maupome, G., et al., Psychological and behavioral acculturation in a social network of Mexican Americans in the United States and use of dental services. Community Dentistry and Oral Epidemiology, 2016. 44(6): p. 540-548.

20. Horn, S.R. and A. Feder, Understanding Resilience and Preventing and Treating PTSD. Harvard Review of Psychiatry, 2018. 26(3): p. 158-174.

21. Carrillo-Diaz, M., et al., Dental fear-related cognitive vulnerability perceptions, dental prevention beliefs, dental visiting, and caries: a cross-sectional study in Madrid (Spain). Community Dentistry and Oral Epidemiology, 2015. 43(4): p. 375-384.

22. Lam, W.W.T., et al., Measuring and Validating a General Cancer Predisposition Perception Scale: An Adaptation of the Revised-IPQ-Genetic Predisposition Scale. Plos One, 2015. 10(11).

23. Azadi, Y., M. Yazdanpanah, and H. Mahmoudi, Understanding smallholder farmers' adaptation behaviors through climate change beliefs, risk perception, trust, and psychological distance: Evidence from wheat growers in Iran. Journal of Environmental Management, 2019. 250.

24. Schwarzer, R., Self-Efficacy in the Adoption and Maintenance of Health Behaviors: Theoretical Approaches and a New Model. Self-efficacy: Thought control of action, 1992.

25. Grothmann, T. and A. Patt, Adaptive capacity and human cognition: The process of individual adaptation to climate change. Global Environmental Change, 2005. 15(3): p. 199-213.

26. Nater, U.M., et al., Coping styles in people with chronic fatigue syndrome identified from the general population of Wichita, KS. J Psychosom Res, 2006. 60(6): p. 567-73.

27. Leung, C., et al., Evaluation of the Chinese version of the Hospital Anxiety and Depression Scale. International journal of psychosomatics : official publication of the International Psychosomatics Institute, 1993. 40: p. 29-34.

28. Arving, C., et al., Early rehabilitation of cancer patientsAn individual randomized stepped-care stressmanagement intervention. Psycho-Oncology, 2019. 28(2): p. 301-308.

29. Schneider, S.S. and J. Bengel, Psychological stress and risk perception of rescue personnel of the Technisches Hilfswerk. Notfall \& Rettungsmedizin, 2014. 17(8): p. 690-696. 
30. Armas, I., R.Z. Cretu, and R. Ionescu, Self-efficacy, stress, and locus of control: The psychology of earthquake risk perception in Bucharest, Romania. International Journal of Disaster Risk Reduction, 2017. 22: p. 71-76.

31. Bates, K., et al., Psychological adaptation to molecular autopsy findings following sudden cardiac death in the young. Genetics in Medicine, 2019. 21(6): p. 1452-1456.

32. Rodriguez, C., et al., Does risk for bipolar disorder heighten the disconnect between objective and subjective appraisals of cognition? Journal of Affective Disorders, 2013. 148(2-3): p. 400-405.

33. Torres-Ortuno, A., et al., Coping strategies in young and adult haemophilia patients: A tool for the adaptation to the disease. Haemophilia, 2019. 25(3): p. 392-397.

\section{Figures}

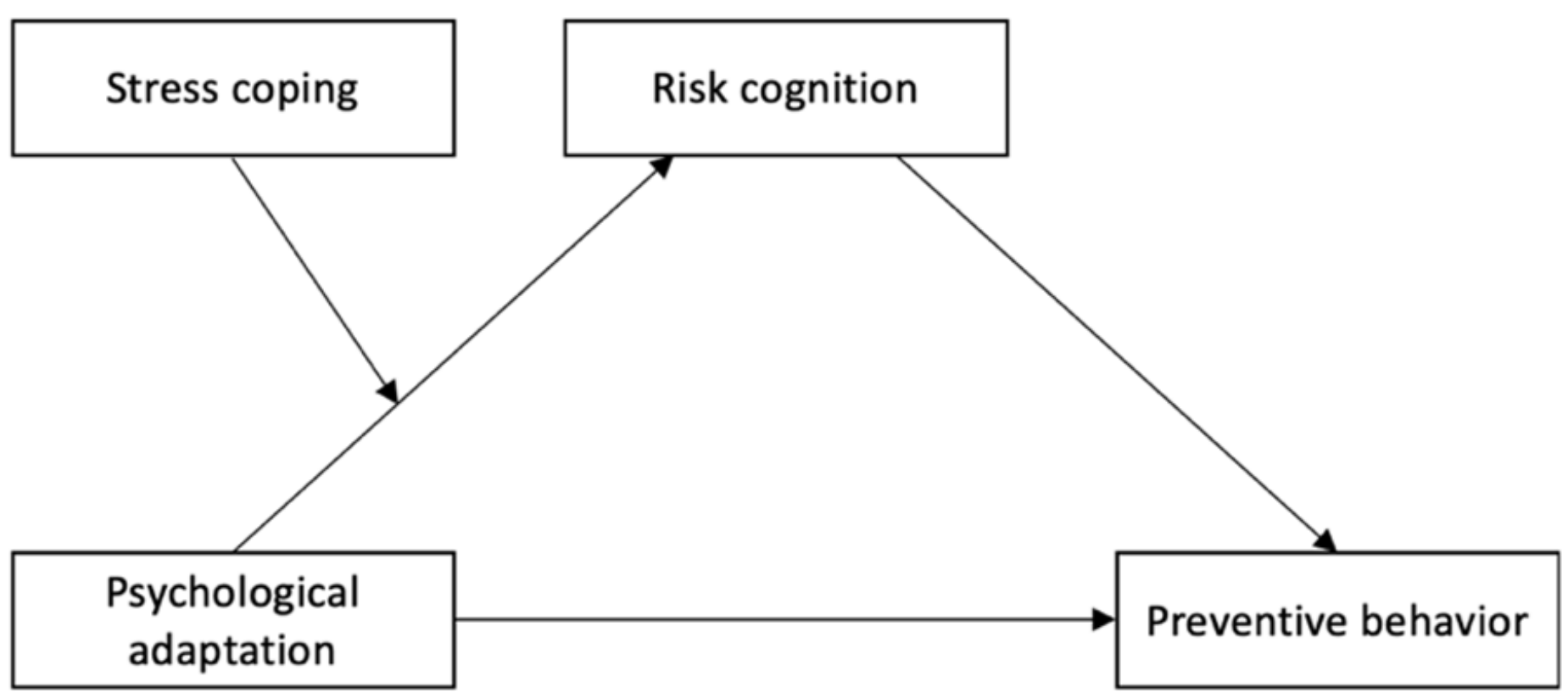

Figure 1

Research Framework 


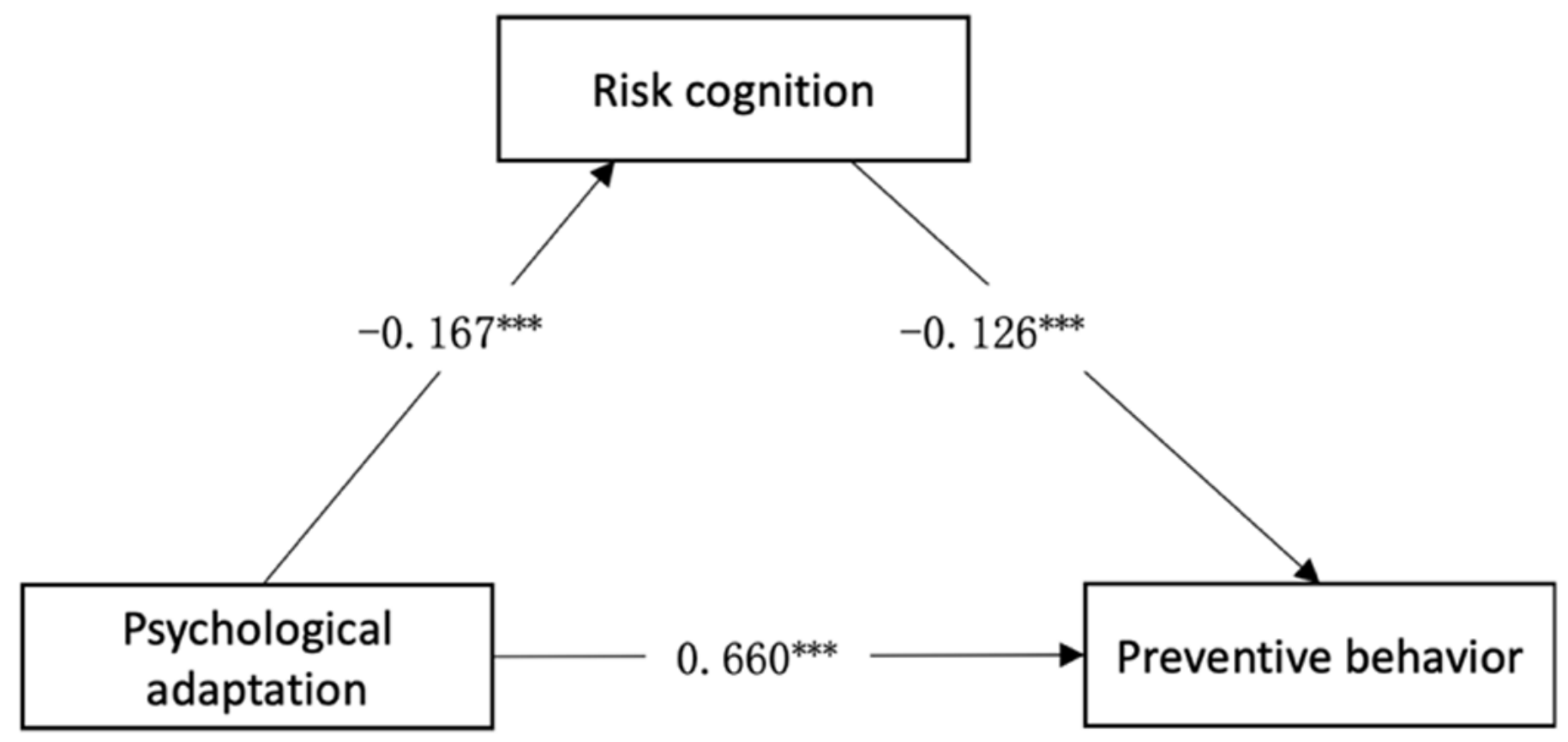

Figure 2

Mediating effect of risk cognition between psychological adaptation and preventive behavior Note: the data have been standardized. $* * * p<0.001, * p<0.05$.

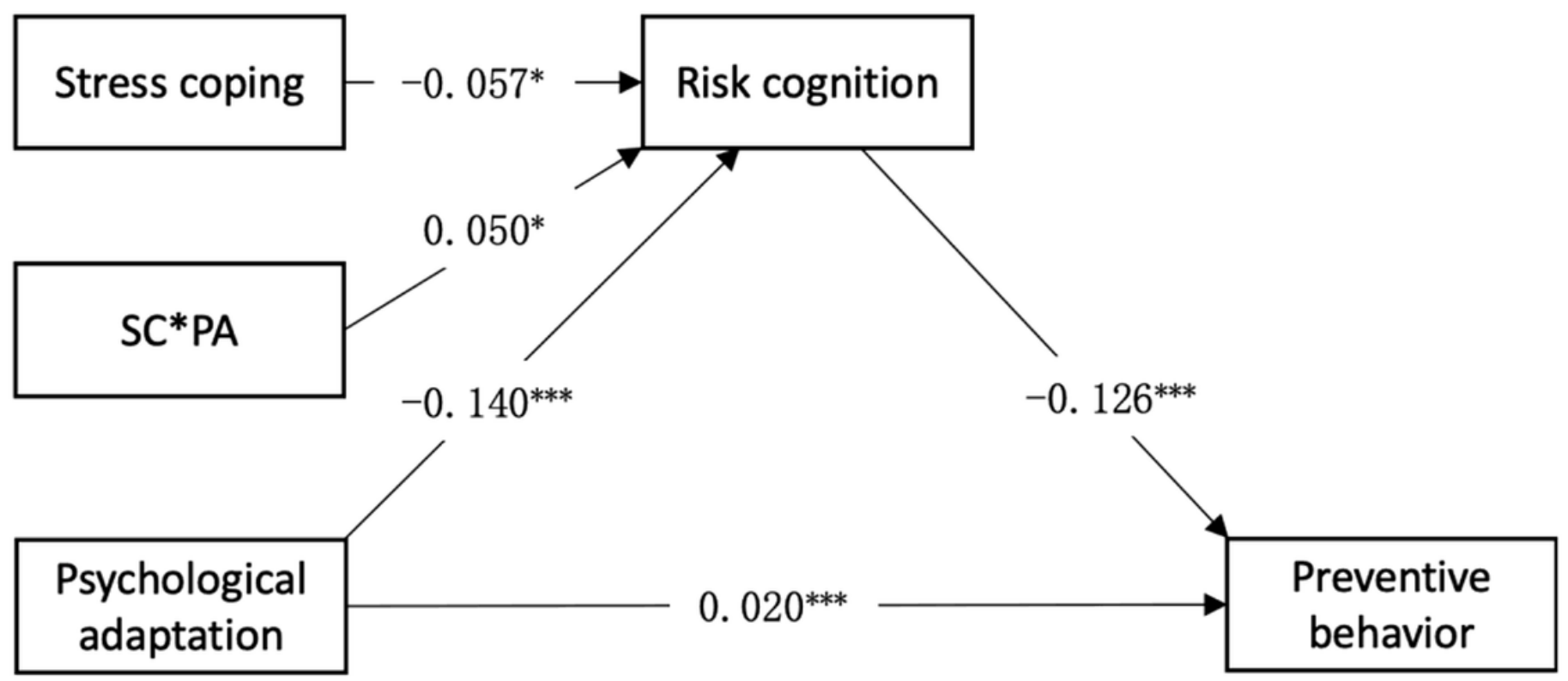

Figure 3

The moderating effect of stress coping on the relationship between psychological adaptation and risk cognition Note: the data have been standardized. ${ }^{* \star} p<0.001,{ }^{*} p<0.05$. PA means psychological adaptation; SC means stress coping. 


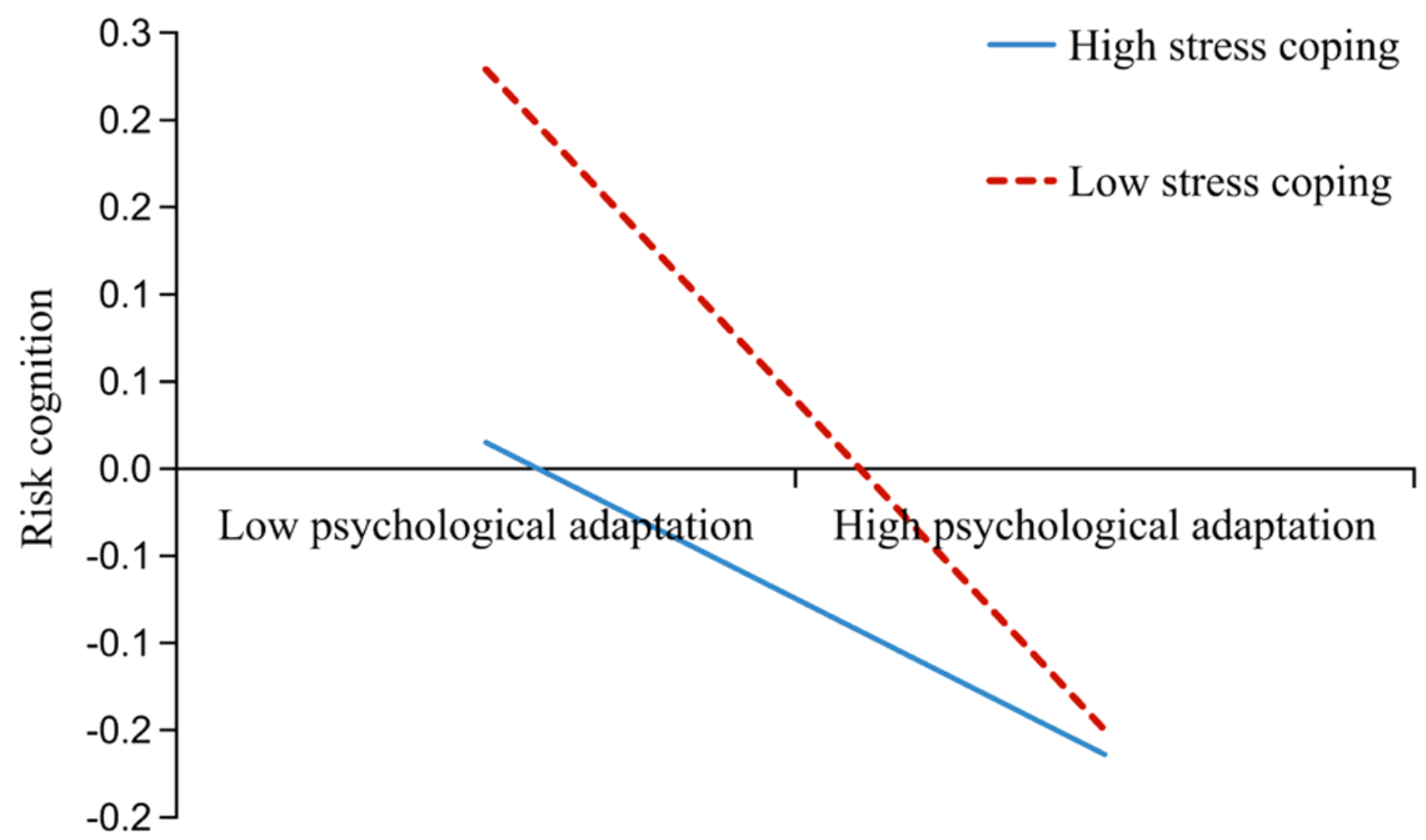

Figure 4

The moderating effect of stress coping on the relationship between psychological adaptation and risk cognition 